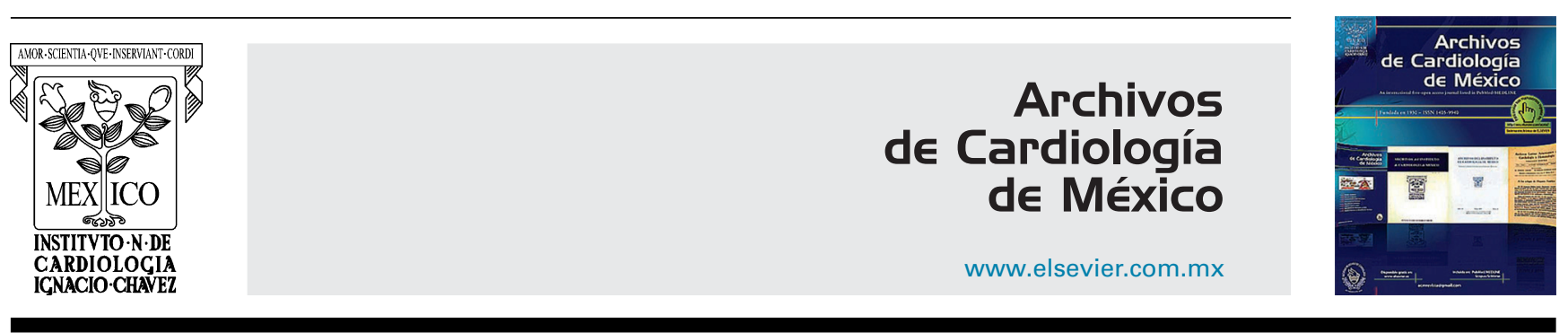

INVESTIGACIÓN CLÍNICA

\title{
Manifestaciones cardiacas en la etapa aguda de la enfermedad de Kawasaki en un hospital pediátrico de tercer nivel en la Ciudad de México
}

\author{
Luis Martín Garrido-García ${ }^{a, *}$, Rocío Alejandra Peña-Juárez ${ }^{\mathrm{b}}$ \\ y Marco Antonio Yamazaki-Nakashimada ${ }^{c}$
}

a Servicio de Cardiología, Instituto Nacional de Pediatría, SSA, Ciudad de México, México

b Cardiología Pediátrica, Hospital General de Occidente, SSA, Guadalajara, Jalisco, México

c Servicio de Inmunología, Instituto Nacional de Pediatría, SSA, Ciudad de México, México

Recibido el 6 de octubre de 2017; aceptado el 12 de marzo de 2018

\author{
PALABRAS CLAVE \\ Enfermedad \\ de Kawasaki; \\ Complicaciones \\ cardiacas; \\ Aneurismas \\ coronarios; \\ México
}

\begin{abstract}
Resumen
Objetivo: Describir las manifestaciones cardiacas en la etapa aguda de la enfermedad de Kawasaki en pacientes atendidos en un hospital de tercer nivel de la Ciudad de México, México.

Métodos: Estudio retrospectivo, descriptivo en pacientes con diagnóstico de enfermedad de Kawasaki de agosto de 1995 a diciembre del 2016 en el Instituto Nacional de Pediatría, México. Se estudio la demografía de los pacientes, características clínicas, tratamiento empleado y desarrollo de complicaciones cardiacas en la etapa aguda de la enfermedad.

Resultados: Se estudiaron 508 casos de enfermedad de Kawasaki. La edad media al diagnóstico fue de $37.64 \pm 35.56$ meses. Predominio de pacientes masculinos del $65.4 \%$, con una relación masculino/femenino de 1.88:1. La mayoría de los casos (79.2\%) tuvo una presentación completa. La gammaglobulina intravenosa fue administrada en $92.4 \%$ de los casos.

Veintiocho pacientes $(5.5 \%)$ desarrollaron arritmias, se presentaron cambios en el segmento ST en 29 pacientes $(5.6 \%$ ) y 5 pacientes desarrollaron isquemia miocárdica.

En el ecocardiograma inicial, 51 pacientes (9.9\%) presentaron datos de miocarditis, 72 pacientes $(14 \%)$ datos de pericarditis y 77 casos tuvieron derrame pericárdico (15\%). Se detectaron alteraciones en las arterias coronarias en 169 casos (32.9\%). Cuatro pacientes fallecieron en la etapa aguda de la enfermedad por complicaciones cardiacas de la enfermedad de Kawasaki. Conclusiones: En México cada vez existen más casos de enfermedad de Kawasaki con un alto porcentaje de manifestaciones cardiacas al diagnóstico. Se requiere de un mayor conocimiento
\end{abstract}

\footnotetext{
* Autor para correspondencia. Av. Insurgentes Sur 3700-C. Colonia Insurgentes Cuicuilco, Ciudad de México, México, CP 04530. Correo electrónico: luismartin_garr@hotmail.com (L.M. Garrido-García).
} 
de la enfermedad en México, para poder establecer cuál es la evolución cardiológica de los pacientes en el país.

(C) 2018 Instituto Nacional de Cardiología Ignacio Chávez. Publicado por Masson Doyma México S.A. Este es un artículo Open Access bajo la licencia CC BY-NC-ND (http://creativecommons. org/licenses/by-nc-nd/4.0/).

\section{KEYWORDS}

Kawasaki disease;

Cardiac

complications;

Coronary artery

aneurysms;

Mexico

\section{Introducción}

La enfermedad de Kawasaki (EK) es una vasculitis sistémica aguda, con un curso determinado y de etiología desconocida. El $80 \%$ de los pacientes afectados son menores de 5 años. Esta enfermedad fue descrita por primera vez en Japón en 1967 por Tomisaku Kawasaki. Actualmente se sabe que tiene una distribución mundial, aunque predomina en Asia y en niños de ascendencia asiática. Se considera que es la principal causa de cardiopatía adquirida en países industrializados ${ }^{1}$. La EK clínicamente se caracteriza por la presencia de fiebre, conjuntivitis no supurativa bilateral, eritema de labios y de la mucosa oral, alteraciones en las extremidades, exantema y adenopatía cervical. La complicación cardiaca más importante es el desarrollo de lesiones coronarias, las cuales se presentan en 15 a $25 \%$ de los casos no tratados de la enfermedad. Estos pacientes tienen un riesgo elevado de desarrollar isquemia miocárdica. El tratamiento de la EK en la etapa aguda está dirigido a reducir la inflamación de la vasculatura coronaria y a prevenir la trombosis coronaria, mientras que el tratamiento a largo plazo en aquellos pacientes que desarrollaron lesiones coronarias está encaminado a la prevención de isquemia o infarto de miocardio ${ }^{1,2}$.

En México existen reportes aislados de la EK, en los cuales se presenta mayor afectación cardiaca que lo reportado en la literatura ${ }^{3-7}$.

El objetivo de este trabajo es describir las manifestaciones cardiacas de la etapa aguda de la EK en pacientes atendidos en un hospital de tercer nivel de la Ciudad de México.

\section{Métodos}

Estudio retrospectivo, descriptivo. Se revisaron los expedientes clínicos de los pacientes con EK de agosto de 1995 a diciembre del 2016 con el diagnóstico de EK en el Instituto Nacional de Pediatría. Para el diagnóstico de la EK se utilizaron los criterios descritos por la Asociación Americana del Corazón ${ }^{1}$. La forma completa se diagnosticó con la presencia de fiebre de más de 5 días de evolución y con 4 de los 5 criterios clínicos principales: eritema polimorfo, hiperemia conjuntival no purulenta, adenomegalia cervical, cambios en las extremidades y lesiones 
orales. Se describieron además las formas incompletas y las presentaciones atípicas de la EK.

Se analizó la edad, sexo, manifestaciones clínicas, tiempo de inicio de la fiebre al diagnóstico de la enfermedad, tratamiento empleado, las manifestaciones cardiacas y la evolución en la etapa aguda de la enfermedad. Se realizó electrocardiograma al diagnóstico de la EK. El ecocardiograma fue realizado el mismo día del diagnóstico de la EK previo a la administración de la gammaglobulina intravenosa por un cardiólogo pediatra con experiencia en la visualización de las arterias coronarias. El estudio se realizó siguiendo los criterios recomendados por la Asociación Americana del Corazón en las siguientes modalidades: modo $M$, bidimensional, Doppler espectral y Doppler color, y se registró el tamaño de las cavidades y la función ventricular, insuficiencia valvular, engrosamiento pericárdico, presencia de derrame pericárdico y se realizó la medición de las arterias coronarias principales ${ }^{1}$. (De agosto de 1995 a diciembre del 2004 se midió en forma sistemática únicamente el tronco de la coronaria izquierda y la coronaria derecha, y posterior al 2005, se midieron todas las arterias coronarias disponibles). Para determinar alteraciones coronarias se midió el diámetro interno máximo del tronco de la coronaria izquierda y de la coronaria derecha y de la descendente anterior y circunfleja en caso de contarse con la medición. Para la determinación de lesiones coronarias desde 1995 hasta el 2004, se utilizaron los criterios de la Asociación Americana del Corazón y posteriormente se realizó estandarización de acuerdo con la superficie corporal. En forma retrospectiva, se determinó la superficie corporal de los pacientes utilizando peso y talla por la fórmula de Haycock ${ }^{8}$. Se realizó una estandarización en todos los casos por la fórmula propuesta por Dallaire ${ }^{9}$. Finalmente, se clasificó el tamaño de las coronarias de acuerdo con lo propuesto por Manlhiot. Se determinó como un tamaño coronario normal si el z-score de las coronarias fue < de 2.5, como aneurisma coronario pequeño, si el z-score fue entre 2.5 y 5 , aneurisma coronario grande si el z-score fue entre 5 y 10 y aneurisma coronario gigante si el z-score fue $\geq 10^{10}$.

Los resultados fueron reportados como media y desviación estándar para las variables cuantitativas y como porcentaje para las variables cualitativas.

\section{Resultados}

Durante el período de estudio se diagnosticaron 514 casos de EK de los cuales se eliminaron 8 casos por no contar con peso y talla para el cálculo de la superficie corporal, por lo que se analizaron 508 casos. La edad media al diagnóstico fue de $37.64 \pm 35.56$ meses con un rango de 2-200 meses. De nuestros casos, 332 fueron masculinos $(65.4 \%)$ con una relación masculino/femenino de 1.88:1. El tiempo de inicio de la fiebre al diagnóstico de la enfermedad fue de $8.98 \pm 5.80$ días con un rango de 3-40 días. De nuestros pacientes, 107 fueron diagnosticados como incompletos (20.8\%). Como complicación cardiológica al diagnóstico de la EK, 6 pacientes presentaron insuficiencia cardiaca (1.2\%) y 17 pacientes choque asociado a EK (3.3\%).

En cuanto al tratamiento, 475 pacientes recibieron gammaglobulina intravenosa en la etapa aguda de la enfermedad (93.5\%). Nueve pacientes recibieron gammaglobulina a dosis
Tabla 1 Clasificación del tamaño de las arterias coronarias en la etapa aguda de la enfermedad de Kawasaki de acuerdo a z-score. 8

\begin{tabular}{lllll}
\hline & $\mathrm{TCl}$ & $\mathrm{DA}$ & $\mathrm{Cx}$ & $\mathrm{CD}$ \\
\cline { 2 - 5 } & $\mathrm{n}=508$ & $\mathrm{n}=268$ & $\mathrm{n}=245$ & $\mathrm{n}=508$ \\
\hline Coronaria normal & 375 & 223 & 222 & 404 \\
Aneurisma pequeño & 83 & 24 & 12 & 56 \\
Aneurisma grande & 30 & 9 & 7 & 22 \\
Aneurisma gigante & 20 & 12 & 4 & 26 \\
\hline
\end{tabular}

CD: arteria coronaria derecha; Cx: arteria circunfleja; DA: arteria descendente anterior; $\mathrm{TCl}$ : tronco coronaria izquierda. Fuente: Haycock et al. ${ }^{8}$.

de $400 \mathrm{~g} / \mathrm{kg}$ de peso por 5 días, y a partir de 1999, todos los pacientes recibieron gammaglobulina en dosis única de $2 \mathrm{~g} / \mathrm{kg}$ de peso.

En el electrocardiograma de superficie al ingreso, 28 pacientes presentaron arritmias (5.5\%), 26 pacientes taquicardia sinusal no relacionada con la fiebre, un paciente con extrasístoles ventriculares monomórficas y un paciente presentó bloqueo AV de primer grado. Se presentaron alteraciones en el segmento S-T (segmento ST aplanado, ondas T negativas o elevación del segmento ST en las derivaciones precordiales) en 29 pacientes $(5.6 \%)$ y 5 pacientes presentaron datos francos de isquemia miocárdica. En todos los casos donde se presentó la isquemia, la lesión fue corroborada mediante un gammagrama cardiaco perfusorio.

En el ecocardiograma al diagnóstico de la EK, 51 pacientes $(9.9 \%)$ presentaron datos de miocarditis, 72 pacientes (14\%) presentaron reforzamiento pericárdico y 77 casos presentaron derrame pericárdico (15\%), requiriendo la realización de pericardiocentesis únicamente un paciente.

En cuanto a la afectación coronaria, se detectaron alteraciones en 169 casos (33.3\%) de las cuales 134 fueron en la arteria coronaria izquierda y 105 casos en la arteria coronaria derecha. En la etapa aguda se encontró alteración en una sola arteria coronaria en 77 casos (15.1\%), en 2 arterias coronarias en 63 casos (12.4\%), en 3 arterias coronarias en 14 casos $(2.8 \%)$ y en 4 arterias coronarias en 15 casos $(2.9 \%)$

La clasificación de acuerdo al tamaño de cada arteria coronaria afectada se muestra en la tabla 1.

En 32 casos se diagnosticó aneurismas gigantes (zscore $\geq 100 \geq 8 \mathrm{~mm}$ ) en la etapa aguda de la enfermedad (fig. 1). En los casos de aneurismas gigantes, en 17 pacientes las lesiones afectaron tanto la coronaria izquierda como la derecha; en 12 casos se afectó únicamente la coronaria izquierda y en solo 3 casos la coronaria derecha fue la única

Tabla 2 Arterias coronarias afectadas con aneurismas gigantes en la etapa aguda de la enfermedad de Kawasaki

\begin{tabular}{llll}
$\begin{array}{l}\text { Aneurismas gigantes al diagnóstico de EK } \\
\mathrm{n}=32 \text { casos }\end{array}$ \\
\hline $\mathrm{TCl}$ & $\mathrm{DA}$ & $\mathrm{Cx}$ & $\mathrm{CD}$ \\
\hline $\mathrm{n}=508$ & $\mathrm{n}=268$ & $\mathrm{n}=245$ & $\mathrm{n}=508$ \\
\hline 20 & 12 & 4 & 22 \\
\hline
\end{tabular}

CD: arteria coronaria derecha; Cx: arteria circunfleja; DA: arteria descendente anterior; $\mathrm{TCl}$ : tronco coronaria izquierda. 


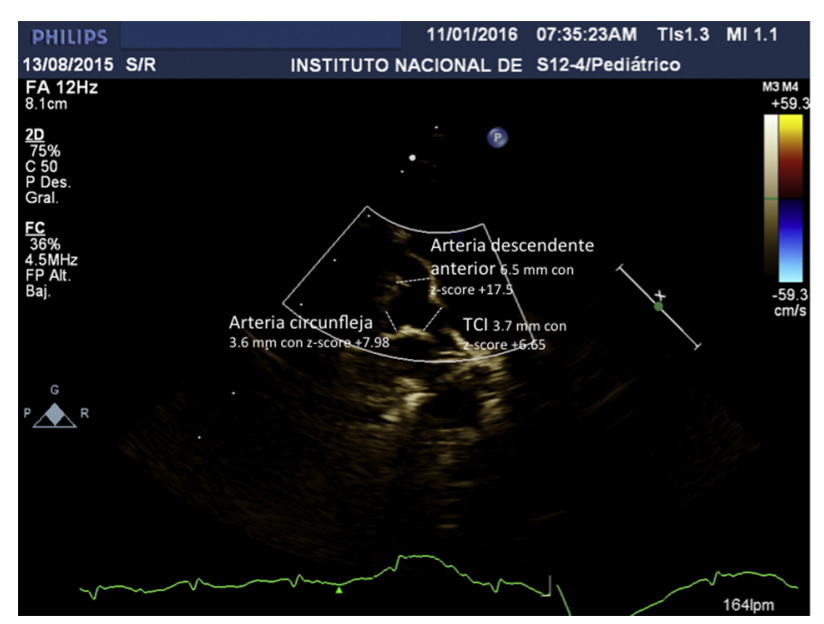

Figura 1 Ecocardiograma en proyección eje largo modificado en un paciente de 4 meses con diagnóstico de enfermedad de Kawasaki, que muestra ectasia grande en el tronco de la arteria coronaria izquierda, ectasia gigante de la arteria descendente anterior y ectasia grande de la arteria circunfleja con un peso de $6.5 \mathrm{~kg}$ y talla $61.5 \mathrm{~cm} \mathrm{SC} 0.34 \mathrm{~m}^{2}$ (Haycock).

arteria afectada. La presentación completa de las arterias afectadas en los casos de aneurismas gigantes se muestra en la tabla 2.

Cuatro pacientes fallecieron en la etapa aguda de la enfermedad por complicaciones cardiacas de la EK (choque 2 pacientes e infarto agudo de miocardio 2 pacientes).

\section{Discusión}

La EK en la actualidad es la segunda vasculitis más frecuente en pediatría tras la púrpura de Henoch-Schönlein ${ }^{11,12}$ y ha reemplazado a la fiebre reumática como la principal causa de cardiopatía adquirida en los niños en países desarrollados $^{13}$. Japón es el país con mayor incidencia de la enfermedad con 265 casos por cada 100,000 menores de 5 años $^{14}$, mientras que la incidencia de la EK en Estados Unidos depende de la raza, siendo más alta en los pacientes de ascendencia asiática con 32.5-39 por cada 100,000 menores de 5 años, de 16.9-19.7 por cada 100,000 niños menores de 5 años en afroamericanos, de 11.1-15.7 por cada 100,000 niños menores de 5 años en hispanos y de 9.1-12 por cada 100,000 niños menores de 5 años en población caucásica ${ }^{15}$. La incidencia de $\mathrm{EK}$ en países europeos recientes es alrededor de 5-10 por 100,000 niños menores de 5 años $^{16}$.

En México, a pesar de que se ha incrementado el número de pacientes diagnosticados con EK, hasta el momento no se cuenta con una casuística real de la enfermedad ya que sigue siendo una enfermedad subdiagnosticada. De acuerdo a los datos obtenidos de la Dirección General de Información en Salud de la Secretaría de Salud existe un incremento significativo en el número de casos de EK diagnosticados en los últimos años ${ }^{17}$ (fig. 2).

En cuanto a las manifestaciones cardiovasculares, en la etapa aguda de la EK se puede afectar cualquier segmento del corazón, desde el endocardio hasta las arterias coronarias $^{18}$. Las manifestaciones cardiovasculares constituyen la principal causa de morbimortalidad asociadas a la EK tanto en la etapa aguda como a largo plazo ${ }^{1,2}$.

En cuanto a las manifestaciones clínicas cardiacas, los pacientes con EK pueden presentar un precordio hiperdinámico, ritmo de galope y soplos en presencia de fiebre o anemia ${ }^{1,2,19}$. En casos raros, se puede presentar choque asociado a Kawasaki, el cual es un síndrome clínico caracterizado por hipotensión, inestabilidad hemodinámica, resistencia al tratamiento con gammaglobulina intravenosa y afectación coronaria severa. Gámez-González et al., en el Instituto Nacional de Pediatría, realizaron un estudio en 214 pacientes con EK del 2000 al 2012 y se documentó la presencia de choque asociado a Kawasaki en 11 pacientes (5.14\%) del total de los casos. Estos pacientes presentaron complicaciones severas al compararlos con los pacientes que no manifestaron choque: infarto de miocardio 27 vs. $1 \%$, resistencia al tratamiento con gammaglobulina 60 vs. $12 \%{ }^{20}$. En el presente trabajo, con 5 años más de estudio en la misma población, se diagnosticaron 300 casos más de EK y solo 5 pacientes presentaron choque asociado a Kawasaki. Esta disminución en la frecuencia de choque está relacionada con un diagnóstico más temprano de la enfermedad.

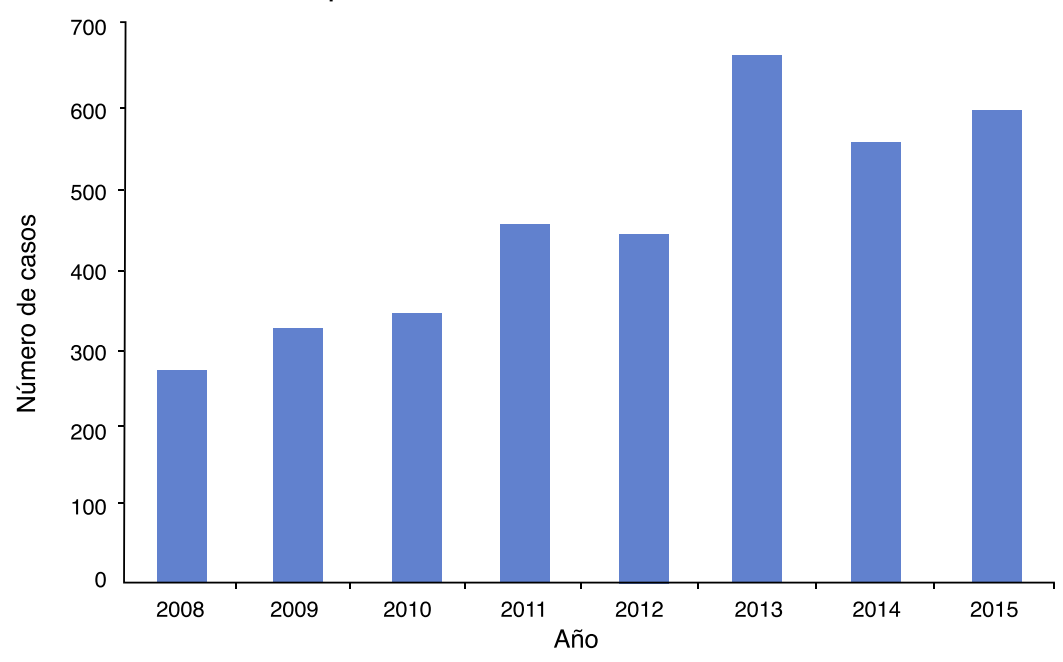

Figura 2 Número de egresos hospitalarios en México en pacientes con diagnóstico de enfermedad de Kawasaki (CIE M303. Síndrome linfomucocutáneo) por año. Fuente: Dirección General de Información en Salud (DGIS). Secretaría de Salud, México ${ }^{17}$. 
De los estudios cardiológicos, en la etapa aguda de la EK, el electrocardiograma puede demostrar cualquier tipo de arritmias incluyendo anomalías de los nodos sinusal y auriculoventricular, con prolongación del intervalo P-R y con cambios inespecíficos en el intervalo ST y alteraciones en la onda $\mathrm{T}$ sobre todo si existe repercusión miocárdica o pericárdica. Puede existir incremento en la dispersión del QT, y también se han reportado anormalidades en la repolarización ventricular. En raras ocasiones se han observado arritmias ventriculares malignas en presencia de miocarditis o isquemia miocárdica ${ }^{21,22}$. En nuestros pacientes se presentó algún tipo de arritmia en solo $5.5 \%$ de los casos, siendo la más frecuente una frecuencia cardiaca mayor a los esperado por la edad y la fiebre; con un solo caso extrasístoles ventriculares y bloqueo ariculoventricular de primer grado, en ambos casos sin repercusión hemodinámica. Las alteraciones en la repolarización en nuestros pacientes se presentaron en casos con aneurismas gigantes y los datos francos de isquemia en los pacientes con infarto en la etapa aguda de la enfermedad. Como se mencionó anteriormente en todos los casos donde se sospechó isquemia electrocardiográfica, esta fue corroborada por estudios de perfusión.

El ecocardiograma transtorácico es el estudio de elección para la evaluación cardiaca en la etapa aguda de la EK que se bien está enfocado a la evaluación de las arterias coronarias, debe evaluarse en su totalidad el estado del corazón ${ }^{1,14,23}$,

La inflamación miocárdica prácticamente se presenta en todos los casos en la etapa aguda de la EK ${ }^{24,25}$. Esta inflamación puede ser demostrada usando estudios de perfusión avanzada desde un 50 al $70 \%$ de los pacientes en la etapa aguda de la $E^{26}$. En el ecocardiograma la inflamación puede manifestarse por alteraciones en la función ventricular izquierda, con alteraciones en la movilidad segmentaria e insuficiencias valvulares, las cuales en su mayoría son leves ${ }^{19,23}$. En nuestro estudio, el $10 \%$ de los pacientes fueron diagnosticados con miocarditis, sin embargo, al ser un estudio retrospectivo, no todos los pacientes pudieron ser evaluados en forma homogénea. En un inicio se consideró como miocarditis a los pacientes que presentaron insuficiencias valvulares o alteraciones en la función ventricular; posteriormente se utilizó el Doppler tisular, y en forma más reciente, se utilizó el análisis de la deformidad sistólica longitudinal para definir la afectación miocárdica en la etapa aguda de la enfermedad. Con estas técnicas ecocardiográficas actuales podrán detectarse en forma más precisa alteraciones en la función miocárdica en la etapa aguda de la $\mathrm{EK}^{27,28}$.

Otra complicación cardiaca en la etapa aguda en la EK es la afectación pericárdica con la presencia de pericarditis y derrame pericárdico, los cuales pueden estar presentes en el 25-30\% de los pacientes con EK y se asocian frecuentemente al grado de vasculitis y miocarditis ${ }^{23,29,30}$. En nuestros pacientes la presencia de pericarditis y/o derrame pericárdico se presentó en el $15 \%$ de los casos y, al igual que lo reportado en la literatura, en la mayor parte de los casos se resolvió posterior al inicio de la gammaglobulina intravenosa.

Las manifestaciones cardiacas más frecuentes en los pacientes con EK son las lesiones en arterias coronarias. Actualmente, las lesiones descritas en las arterias de estos pacientes son como ectasias o aneurismas ${ }^{23}$ (fig. 3). Estas lesiones coronarias se presentan en el $15-25 \%$ de todos los

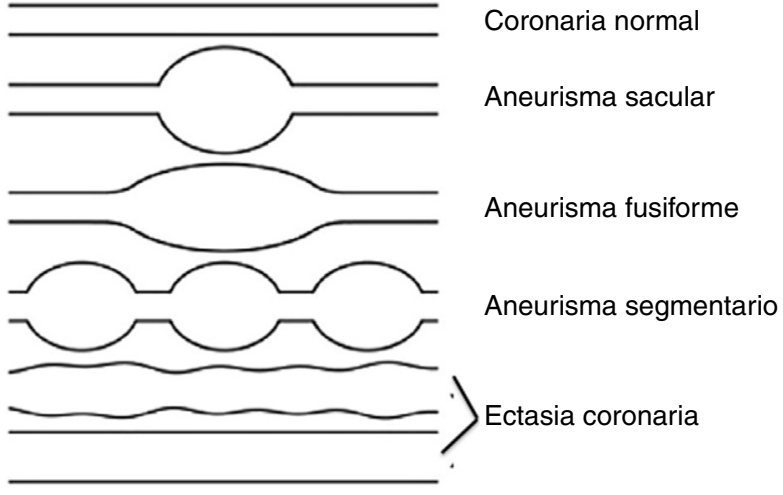

Figura 3 Esquemas de las alteraciones coronarias en la enfermedad de Kawasaki. Modificado de Yim et al. ${ }^{19}$.

pacientes no tratados pero, de acuerdo a los consensos japoneses o americanos, este riesgo disminuye hasta el $5 \%$ en los pacientes tratados con gammaglobulina intravenosa ${ }^{1,2}$. En nuestros pacientes se encontró una mayor frecuencia de lesiones coronarias al diagnóstico que lo reportado en la literatura, sin embargo en estudios de EK en Latinoamérica (REKAMLATINA) se ha observado la misma frecuencia de lesiones coronarias, por lo que se requieren mayores estudios para tratar de establecer la causa de este incremento en patología coronaria en México y Latinoamérica ${ }^{31-33}$.

Es importante señalar que la definición de anomalía coronaria en la EK se ha modificado, desde le determinación por tamaño propuesta por la Academia Americana del Corazón hasta la que es utilizada en la actualidad, la cual está basada en el z-score por la superficie corporal. Esta definición ha permitido una mejoría en la clasificación de lesión coronaria, sobre todo en los pacientes más pequeños con $\mathrm{EK}^{8,34}$. Aunque es necesario señalar que, para una correcta visualización de las arterias coronarias, sobre todo en los segmentos más distales, se requiere de una curva de aprendizaje importante que depende del número de pacientes con EK diagnosticados en la etapa aguda por centro hospitalario ${ }^{35,36}$.

\section{Conclusiones}

La EK representa la cardiopatía adquirida más frecuente en países desarrollados y en México cada vez existen más casos diagnosticados de la enfermedad. Hasta nuestro conocimiento, este trabajo representa el mayor número de casos de EK en el país, con un alto porcentaje de manifestaciones cardiacas al diagnóstico. Se requiere de un mayor conocimiento de la enfermedad en México, para poder establecer en realidad cuál es la evolución cardiológica de los pacientes en el país.

\section{Financiación}

Ninguna.

\section{Conflicto de intereses}

Los autores declaran no tener conflicto de intereses. 


\section{Bibliografía}

1. McCrindle BW, Rowley AH, Newburger JW, et al. Diagnosis, treatment and long-term management of Kawasaki disease. A scientific statement for health professionals from the American Heart Association. Circulation. 2017;135:e927-99.

2. Newburger JW, Takahashi M, Burns JC. Kawasaki disease. JACC. 2016;67:1738-49.

3. Sotelo N, González LA. Kawasaki disease: A rare pediatric pathology in Mexico. Twenty cases report from the Hospital Infantil del Estado de Sonora. Arch Cardiol Mex. 2007;77:299-307.

4. Quezada-Chavarría G, Ramírez-Serrallonga R, Quezada-Cuevas SE, et al. Enfermedad de Kawasaki. Análisis de 17 casos. Rev Med Inst Mex Seguro Soc. 2009;47:61-4.

5. Garrido-García LM, Soto-Blanquel JL, Espinosa-Rosales FJ. Enfermedad de Kawasaki: cuadro clínico, exámenes de laboratorio y lesiones coronarias. Acta Pediatr Mex. 2015;36:314-21.

6. Sotelo-Cruz N. A review of Kawasaki disease, a perspective form the articles published in Mexico since January 1977 to May 2012. Arch Cardiol Mex. 2013;83:214-22.

7. García-Rodríguez F, Flores-Pineda AJ, Villarreal-Treviño AV, et al. Enfermedad de Kawasaki en un hospital pediátrico en México. Bol med Hosp Infant Mex. 2016;73:166-73.

8. Haycock GB, Schwartz GJ, Wisotsky DH. Geometric method for measuring body surface area: A height-weight formula validated in infants, children and adults. J Pediatr. 1978;93:62-6.

9. Dallaire F, Dahdah N. New equations and a critical appraisal of coronary artery z-scores in healthy children. J Am Soc Echocardiogr. 2011;24:60-74.

10. Manlhiot C, Millar K, Golding F, et al. Improved classification of coronary artery abnormalities based only on coronary artery z-score after Kawasaki disease. Pediatr Cardiol. 2010;31:242-9.

11. Cimaz R, Sundel R. Atypical and incomplete Kawasaki disease. Best Pract Res Clin Rheumatol. 2009;23:689-97.

12. Sharma D, Singh S. Kawasaki disease: A common childhood vasculitis. Indian J Pediatr. 2015;10:578-83.

13. Uehara R, Belay ED. Epidemiology of Kawasaki disease in Asia, Europe and the United States. J Epidemiol. 2012;22:79-85.

14. Makino N, Nakamura Y, Yahiro M, et al. Descriptive epidemiology of Kawasaki disease in Japan, 2011-2012: From the results of the 22nd nationwide survey. J Epidemiol. 2015;25:239-45.

15. Okubo Y, Nochioka K, Sakakibara H, et al. National survey of pediatric hospitalizations due to Kawasaki disease and coronary artery aneurysms in the USA. Clin Rheumatol. 2017;36:413-9.

16. Dietz SM, van Stijn D, Burgner D, et al. Dissecting Kawasaki disease: A state-of-the-art review. Eur J Pediatr. 2017;176:995-1009.

17. Dirección General de Información en Salud (DGIS). Secretaría de Salud [consultado 10 Ago 2017]. Disponible en: http: / /www. dgis.salud.gob.mx/contenidos/basededatos/BD_Cubos.html.

18. Singh S, Sharma A, Jiao F. Kawasaki disease: Issues in diagnosis and treatment. A Developing country perspective. Indian J Pediatr. 2016;83:140-5.

19. Yim D, Cheung M, Burgner D. An update in Kawasaki disease II. Clinical features, diagnosis, treatment and outcomes. J Pediatr Child Health. 2013;49:614-23.

20. Gámez-González LB, Murata Ch, Muñoz-Ramírez M, et al. Clinical manifestations associated with Kawasaki disease shock syndrome in Mexican children. Eur J Pediatr. 2013;172:337-42.
21. Sumitono N, Kawasawa K, Taniguchi K, et al. Association of sinus node dysfunction, atrioventricular node conduction abnormality and ventricular arrhythmia in patients with Kawasaki disease and coronary involvement. Cir J. 2008;72:274-80.

22. Crystal MA, Syan SK, Yeung RS, et al. Echocardiographic and electrocardiographic trends in children with acute Kawasaki disease. Can J Cardiol. 2008;24:776-80.

23. Yim, D, Burgner D, Cheung $M$. Echocardiography in Kawasaki disease. En: Bajraktari G, editor. Echocardiography in specific diseases. Tech Editors; 2012. p. 139-52.

24. Kuwabara M, Yashiro M, Kotani K, et al. Cardiac lesions and initial laboratory data in Kawasaki disease: A Nationwide Survey in Japan. J Epidemiol. 2015;25:189-93.

25. Aggarwal P, Suri D, Narua N, et al. Symptomatic myocarditis in Kawasaki disease. Indian J Pediatr. 2012;79:813-4.

26. Kao CH, Hsieh KS, Wang YL, et al. The detection of ventricular dysfunction and carditis in children with Kawasaki disease using equilibrium multigated blood pooling ventriculography and 99Tcm-HMPAO-labelled WBC heart scans. Nucl Med Commun. 1993;14:539-43.

27. Martínez-García A, Ruiz-Esparza E, Vázquez Antona C, et al. Deformación sistólica longitudinal del ventrículo izquierdo en pacientes pediátricos con antecedente de enfermedad de Kawasaki. Arch Cardiol Mex. 2016;86:196-202.

28. Dedeoglu R, Barut K, Oztunuc F, et al. Evaluation of myocardial deformation in patients with Kawasaki disease using speckletracking echocardiography during mid-term follow-up. Cardiol Young. 2017;27:1377-85.

29. Gaur L, Waloff K, Schiller O, et al. Non-coronary inflammation in Kawasaki disease is associated with abnormal myocardial deformation in the acute phase. J Am Soc Echocardiogr. 2014;27:1329-35.

30. Lega JC, Bozio A, Cimaz R, et al. Extracoronary echocardiographic findings as predictors of coronary artery lesions in the initial phase of Kawasaki disease. Arch Dis Child. 2013;98:97-102.

31. Salgado A, Ulloa-Gutiérrez R, Estripeaut D, et al., Study Group Members. Multicenter retrospective study of the clinical course of Kawasaki disease in Latin American Children: The Rekamlatina-2 Study. Circulation. 2015;131 Suppl 2:A015.

32. Collia A, Ellis A, Bueno N, et al. Epidemiology, coronary artery lesions and treatment of Kawasaki disease in children from Argentina: period June 2014 to December 2016. 7th World Congress of Pediatric Cardiology and Cardiac Surgery (WCPCCS 2017) Barcelona, Spain. July 16-21, 2017. Poster 2780.

33. Vargas-Gutiérrez M, Li-Chan S, Avila-AGüero ML, et al. Coronary artery lesions in Costa Rican children with Kawasaki disease: a 5year retrospective study (period 2009-2013). 7th World Congress of Pediatric Cardiology and Cardiac Surgery (WCPCCS 2017) Barcelona, Spain. July 16-21, 2017. Poster 2947.

34. Lin MT, Chang CH, Hsieh WC, et al. Coronary diameters in Taiwanese children younger than 6 years old: z-score regression equations derived from body surface area. Acta Cardiol Sin. 2014;30:266-73.

35. Brown LM, Duffy E, Mitchell C, et al. Practical guide to pediatric coronary artery imaging with echocardiography. J Am Soc Echocardiogr. 2015;28:379-91.

36. Margossian R, Lu M, Minich LA, et al. Predictors of coronary artery visualization in Kawasaki disease. J Am Soc Echocardiogr. 2011;24:53-9. 